Mathematical Research Letters 3, 569-585 (1996)

\title{
SOME NEW APPLICATIONS OF GENERAL WALL CROSSING FORMULA, GOMPF'S CONJECTURE AND ITS APPLICATIONS
}

\author{
AI-Ko LiU
}

\section{Introduction}

As early as the birth of Seiberg Witten invariants [W1], the positive scalar curvature metrics on four dimensional manifolds have played a very important role. It was Witten [W] who first noticed that assuming $b_{2}^{+}>1$ then one could easily derive the vanishing result of Seiberg Witten Invariants for those manifolds which carried the positive scalar curvature (psc) metrics. Combined with Taubes' nonvanishing result [T2] for symplectic four manifolds, one could easily conclude that if a symplectic four manifold carries some psc metric, then its $b_{2}^{+}$must be equal to one. In addition the same vanishing result was used by P. Kronheimer and T. Mrowka [K.M.] in proving the Thom conjecture, and by C.H. Taubes in his "more constraints of symplectic forms on $C P^{2}$ " [T3]. Furthermore R. Friedman and J. Morgan $[\mathrm{FM}]$ systematically discussed which kind of Kahler manifold can carry the psc metrics. Based on surface classification theory, Friedman and Morgan could argue that the Kahler surfaces carrying psc metrics are either rational, rational ruled or irrational ruled. Recently, the same vanishing result has been used by Taubes [T4] to show that $C P^{2}$ has a unique symplectic structure. At this point it is interesting to ask about the possibilities of classifying symplectic four manifolds carrying psc metrics. One of the purposes of this paper is to address this possibility.

Another goal of this paper is to address the question raised by Gompf [Gom] which states that:

Conjecture. (Gompf) If $M$ is a minimal symplectic four manifold with $c_{1}(K)^{2}<0$, then it must be symplectomorphic to the irrational ruled surfaces. (where $c_{1}(K)$ means the first chern class of the canonical bundle associated with the almost complex structures on $M)$.

I will satisfy both purposes by providing some new applications of the wall crossing formula for Seiberg-Witten invariants. I will concentrate

Received October 25, 1995. 
on the compact symplectic four manifolds assuming $b_{2}^{+}=1$. A pesudoholomorphic rational curve with non-negative self intersection number can be generated on the appropriate symplectic four manifold under some weak condition; and therefore, various applications can be derived which include the Gompf conjecture and its corollaries.

\section{Main result}

It is important to have an overall view of the main focus of the theorems proved in this paper. The main theorems and corollaries proved in this paper will be listed according to the English characters. The technical lemmas and the propositions will be listed in terms of numbers.

Main Theorem A. Let $M$ be a $b_{2}^{+}=1$ minimal symplectic four manifold, if $c_{1}(K)^{2}<0$, then $M$ must be an irrational ruled manifold.

Combined with C. H. Taubes' theorem [T6],

Theorem. (Taubes) Let be a minimal symplectic four manifold with $b_{2}^{+}>$ 1 , then $c_{1}(K)^{2}$ must be non-negative.

We prove the Gompf's conjecture which states:

Conjecture. (Gompf) Let $M$ be a minimal symplectic four manifold with $c_{1}(K)^{2}<0$. Then it must be irrational ruled.

This theorem should be viewed as a theorem concerning the classification of symplectic four manifolds.

Using the same argument one can deduce the following:

Theorem B. Let $M$ be $a b_{2}^{+}=1$ symplectic four manifold (not necessarily minimal). If $c_{1}(K) \cdot \omega<0$, then it must be either rational, rational ruled or irrational ruled.

One application of the above theorem is that it can be used in proving the following:

Theorem C. Let $M$ be a symplectic four manifold with psc metrics, then $M$ must be either some blow up of rational, rational ruled or irationally ruled surfaces.

In addition, using the above thorem one can prove that

Theorem D. [LL] If $M$ is a symplectic four manifold with $b_{2}^{+}=1$. and if $C \in H^{2}(M)$ is a class such that $C \cdot \omega>0$ and $C^{2}=C \cdot K=0$, then the Poincare dual of $C$ cannot be represented by a square zero sphere.

Using the theorems proved above in combination with Friedman-Morgan's argument of Seiberg-Witten theory on Kahler surfaces [FM], one is able to prove the following: 
Theorem E. [LL3] If $M$ is a minimal symplectic four manifold with $b_{2}^{+}(M)=1$, and if $c_{1}(K)^{2}>0$, then $\pm c_{1}(K)$ are the only Seiberg-Witten basic classes on $M$. If $c_{1}(K)^{2}=0$, then its basic classes are propotional to $c_{1}(K)$ up to torsions.

From Theorem E, the wall crossing formula [LL1], the blow up formula $[\mathrm{FM}]$, and others, we can gain a qualitative understanding of the Seiberg Witten invariants and the Gromov invariants in regards to these unknown symplectic manifolds. The qualitative understanding of these invariants implies that these minimal symplectic manifolds are of simple type in the metric chamber. By the blow-up formula we know that the blown up manifolds are still of simple type in the blown up chamber(for the definition, please consult $[\mathrm{FM}]$ ). Using the wall crossing formula deduced in [LL1], one can show that in most of the cases, they are of Gromov non-simple type. Therefore, it is a very interesting question to ask whether we can manage these ample families of symplectic curves on these manifolds to study the symplectic geometry of these manifolds.

Let us briefly list the basic tools used in the discussion.

The first important tool is Taubes' theorem " $S W=G r$ " [T1,T4,T6]. Taubes argues that if $b_{2}^{+}>1$ and if some class has a non-zero Gromov invariant, then the cohomology class could be represented by a smooth symplectic curve which might not be connected. Nevertheless, the curve must be reduced so that each connected component can only have a multiplicity of one. (Though it holds true in most cases. The symplectic tori can have higher mulitiplicities.) A similar conclusion can be made for the $b_{2}^{+}=1$ symplectic manifolds, however, with an essential difference. To argue that some multiple covering of a -1 curve doesn't appear, Taubes used the blow up formula of $S W$ invariants. It is important to remember that in the blow up formula, the new basic classes would change by $\pm E$, where $E$ is the exceptional class. However, as the $b_{2}^{+}=1$ symplectic manifolds usually are not of simple type, one could argue that there is a similar blow up formula for the corresonding chambers with the coefficients before $\mathrm{E}$ being arbitrary odd integers besides \pm 1 . The only restriction involves making the moduli space dimension of the new "basic class" in the blown up chamber non-negative. Therefore, one can not argue by this process that a multiple covering of a -1 curve does not appear. In fact, if we blow up several points on $C P^{2}$, it is easy to derive some examples where the multiple covering of -1 curves do appear as some connected component of the symplectic curve. To avoid this type of result, one needs to be careful in using Taubes' theorem. In general, there are two cases where one can use Taube's theorem effectively. The first is when the symplectic manifold is itself minimal. The other is when the class of the symplectic curve is 
orthogonal to all the possible exceptional curves. Besides these two cases, we need a detailed discussion. For most of the applications in this paper, either one of the cases can be shown to occur.

The following are the lemmas and the theorems which will be used frequently in the paper.

Lemma 1. (light cone lemma) For the light cone of signature $(1, n)$ $(n \neq 0)$, any two elements in the forward cone have non-negative dot product. Especially, if the dot product is zero then the two elements are propotional to each other.

Theorem 2. (McDuff) If a symplectic four manifold $M$ has a non-negative self intersecting rational curve, then it must be symplectomorphic to either rational, rational ruled, or irrational ruled manifolds.

The other useful tools are the following lemmas proved previously in papers [LL1],[LL2].

Lemma 3. Consider the $\operatorname{spin}_{c}$ structure associated with $c_{1}\left(K^{-1}\right)+2 C$. If $\left(c_{1}\left(K^{-1}\right)+2 C\right) \cdot \omega>0$, then there is an odd number of walls crossed when deforming from the honest $S W$ equation into the final Taubes' chamber. Conversely if the sign is negative, there is an even number of walls crossed. (where $\omega$ is the sympletic form used to define the deformation of the SeibergWitten equation.)

Lemma 4. If a symplectic four manifold carries positive scalar curvature metrics, then $c_{1}\left(K^{-1}\right) \cdot \omega_{\text {psc }}$ is always greater than zero.

$\omega_{p s c}$ here means the self-dual harmonic 2 -form associated to the positive scalar curvature metric which lies in the forward light cone. This lemma is nothing but Taubes' "more constraints" on symplectic forms for $C P^{2}[\mathrm{~T}]$. Over here it is written in a much more general form.

To find the appropriated cohomology class for the latter usuage, one needs the following arithematic lemma.

Lemma 5. If $q$ is an integral indefinite quadratic unimodular form of signature of type $\left(1, b_{2}^{-}\right)$(require $\left.b_{2}^{-} \neq 0\right)$, then the rational points are dense in the real locus of the light cone defined by $q=0$.

The proof is done by using the Hasse-Minkowski classification of the indefinite unimodular form [Ser], then one can directly write down a rational solution of $q=0$. If $q$ is of even type, one can get the rational solution directly by restricting to the hyperbolic factor. The odd type case can be handled easily. With the first rational point being found, it is elementary to show that the conic (in $P^{b_{-}}(R)$ ) associated with the light cone by 
projectivization is birational to $P^{\left(b_{-}-1\right)}(R)$ over $Q$ (this is where the first rational point is used). This proves the denseness of the rational points.

An immediate corollary is the following.

Proposition 6. Let $c_{1}(K)$ be an element in $H^{2}(M, Z)$ such that $c_{1}(K)^{2}<$ 0 . Then there exists an integral element $Z$ of the forward light cone $(q=0)$ such that $c_{1}(K) \cdot Z<0$.

Proof. It is easy. If $c_{1}(K)^{2}<0$, then there exists some real element $x$ of the forward light cone $q=0$ such that $c_{1}(K) \cdot x<0$. Now use the denseness of the rational points, one can assume that $x$ itself is rational. By rescaling, if necessary, one can definitely make $x$ integral. From now on let us call this the element $Z$.

The usage of the Lemmas 5 and 6 here are a tangent from Wilson's paper [Wil].

Let us begin our discussion now. Suppose $M$ is a symplectic four manifold satisfying the hypothesis of the Gompf's conjecture, the following proposition forces the manifold $M$ to have a non-zero first Betti number.

Proposition 7. If $M$ is a minimal symplectic four manifold with $c_{1}(K)^{2}<$ 0 , then its first Betti number $b_{1}$ must be non-zero.

Proof. Suppose that $b_{1}$ is actually zero, let us derive a contradiction. Notice that in this case Kronheimer-Mrowka's wall crossing formula [K.M.]can be used and the wall crossing number is always \pm 1 if the dimension of the moduli space is non-negative.

Instead of choosing only one cohomology class, let us begin by using a family of them. Let $k \cdot Z$ (where $Z$ was defined by the previous proposition, and $k \in Q$.) be such a family.

Then we notice that for these classes, the Gromov(and also the Seiberg Witten) moduli space dimension is

$$
(k \cdot Z)^{2}-c_{1}(K) \cdot(k Z)=-k c_{1}(K) \cdot Z>0 .
$$

And also the adjunction formula gives us

$$
(k \cdot Z)^{2}+c_{1}(K) \cdot(k Z)<0 .
$$

Because our manifold $M$ is minimal, the problem of multi covering of -1 curves never shows up. Therefore it is safe to use Taubes' theorem " $G r=S W "$ now.

The claim is the following: For these classes $k \cdot Z$ (where $\mathrm{k}$ is greater than 0$), \operatorname{Gr}(k \cdot Z)$ are all zero. The reason is as follows. If one of them has 
non-zero Gromov invariant, then $k_{0} \cdot Z$ must be represented by a symplectic curve. Let us denote cohomologically

$$
k \cdot Z=\sum C_{i}
$$

with all $C_{i}$ disjoint to each other. Plugging this into the adjunction formula we get

$(k \cdot Z)^{2}+c_{1}(K) \cdot(k \cdot Z)=\sum\left(C_{i} \cdot C_{i}+c_{1}(K) \cdot C_{i}\right)=\sum\left(2 g\left(C_{i}\right)-2\right)<0$.

However as the adjunction number is negative, a symplectic sphere must be in the list of the connected components (otherwise each $2 g-2$ is always nonnegative if genus is bigger or equal to one). Having assumed $M$ is minimal ; therefore, this rational curve cannot be a -1 symplectic sphere. Then it is forced to be a rational curve with non-negative self intersection number! Now apply McDuff's theorem [M] to conclude that this minimal model $M$ must be either $P^{2}$ or $P^{1} \times P^{1}$ having $c_{1}(K)^{2}>0$ (using $c_{1}(K)^{2}=2 \chi+$ $3 \sigma)$ and therefore contradicts to our original assumption. Using Taubes' theorem " $S W=G r$ " we conclude that $S W\left(c_{1}\left(K^{-1}\right)+2 k Z\right)$ are all zero in Taubes' chamber. The speciality of these classes is that they actually form a ray. Now let us focus our attention to the following number

$$
\left(c_{1}\left(K^{-1}\right)+2 k Z\right) \cdot \omega
$$

where $\omega$ is the symplectic form of the symplectic manifold $M$. As $Z$ is chosen to be in the boundary of the forward light cone and $\omega$ is in the interior, the cup product of $k Z$ and $\omega$ must be strictly positive by the light cone lemma.

The above formula, when being viewed as an affine function in $k$, has a positive leading term. This implies that the value will be positive for large enough $k$. However when deforming backwards beginning from Taubes' chamber, the $\operatorname{spin}_{c}$ structure $c_{1}\left(K^{-1}\right)+2 k Z$, the number of walls crossed in the two steps is in fact odd for large enough $\mathrm{k}$. Therefore this implies (by using the wall crossing formula of Kronheimer and Mrowka) that in the ordinary metric chamber, $S W\left(c_{1}\left(K^{-1}\right)+2 k Z\right)$ all are non-zero for large enough $k$. This is impossible because it is well known that for a given generic metric and self dual two form $\mu$ as perturbation, there can be only finite number of $\operatorname{spin}_{c}$ structures having non-zero Seiberg-Witten invariants! This creates a contradition and therefore ends the proof.

Now assume that $b_{1}$ is non-zero and the general wall crossing formula can be used. Before I proceed, we rule out the following: 
Lemma 8. Let $M$ be a minimal symplectic four manifold with $c_{1}(K)^{2}<0$ and $b_{1}>0$. Then it must satisfy the following condition. For all non-zero $y \in H^{1}(M, R)$, the map

$$
y \cup \cdot: H^{1}(M, R) \longrightarrow H^{2}(M, R)
$$

must be non-zero.

The proof is done by mimicing Taubes' theorem [T1] listed above.

Proof. Suppose that the above conclusion does not hold. Then it is easy to see that for this hypothetical manifold, the wall crossing number calculated

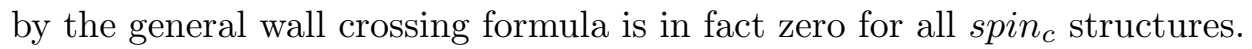
This point has an important implication. Namely, even though this manifold is of $b_{2}^{+}=1$ type and the walls exist, the $S W$ invariants do not jump after crossing any wall. In particular this implies that the Seiberg Witten invariants are diffeomorphism invariants!

Now by applying Taubes' existence theorem for $S W\left(K^{-1}\right)$ [T2], one can conclude that for both $K$ and $K^{-1}$, their $S W$ invariants, no matter in which chamber, are both \pm 1 . Using this fact in the Taubes' chamber and writing $K$ as $K^{-1}+2 K$, we find that, in the $b_{2}^{+}>1$ case, the class $c_{1}(K)$ can be represented by a symplectic curve [T1]. Adjunction formula consideration will force the curve to contain a non-negative self intersecting rational curve as a connected component (note: the minimality is enforced). Now McDuff's theorem implies that $M$ must be irrational ruled. This causes a contradiction because we know explicitly that on irrational ruled surfaces, the cup product map defined above is always non-trivial.

Therefore, in the following discussion the cup product map is always assumed to be non-zero. Before continuing onto the main argument, I would like to address the following lemmas:

Lemma 9. Let $M$ be a $b_{2}^{+}=1$ symplectic four manifold. Assuming $b_{1} \neq 0$ and that every cup product map defined above is non-zero, then there always exists an integral symplectic basis of $H^{1}(M, R)$ which is nondegenerate.

Proof. Using the fact that

$$
y_{1} \cdot y_{2} \cdot y_{3} \cdot y_{4}=0
$$

for every four elements in $H^{1}(M, R)$ and combining with the light cone lemma, we learn that the image of the cup product $\cdot \cup \cdot: H^{1} \times H^{1} \longrightarrow H^{2}$ must form a line lying in the boundary of the light cone. Let us call the primitive generator of that line $C$. We can choose $C$ to be in the forward light cone. Then the cup product on $H^{1}$ can be viewed as a quadratic form 
defined by the coefficient in front of $C$. The fact that the cup product maps defined in the previous lemma are all non-zero immediately implies that this quadratic form is nondegenerate and symplectic. Thus the symplectic basis exists and we know that:

Corollary 10. [LL] A spinc structure $\mathcal{L}$ (with non-negative moduli space dimension) has a non-zero wall crossing number if $c_{1}(\mathcal{L}) \cdot C \neq 0$.

Now it is time to prove the main theorem.

Theorem A. Let $M$ be a minimal symplectic four manifold with $c_{1}(K)^{2}<$ 0 , then $M$ must be irrational ruled.

Proof. On the contrary, suppose that $M$ is not irrational ruled. We can argue that $\operatorname{Gr}(k Z)=0$ for all classes $k \cdot Z$ ( where $k>0)$. Using Taubes' theorem " $G r=S W$ ", it implies that for all these classes, $S W\left(c_{1}\left(K^{-1}\right)+\right.$ $2 k Z)$ are zero. For large enough $k$, the number of walls crossed in the step 2 and 3 (for the details, please consult [LL2]) is odd. On the other hand we know that in the ordinary metric chamber there can be only a finite number of non-zero Seiberg Witten classes. Thus we conclude that the wall crossing number for $c_{1}\left(K^{-1}\right)+2 k Z$ must be zero for large enough $k$. Using Corollary 10 this statement can be rephrased as the following equation:

$$
\left(c_{1}\left(K^{-1}\right)+2 k Z\right) \cdot C=0, k \gg 0 .
$$

The LHS is an affine function in the variable $k$. The fact that it has an infinite number of zero forces both the leading term and the constant term to be zero. That is to say

$$
c_{1}(K) \cdot C=0, Z \cdot C=0 .
$$

One can easily see that these two equations contradict each other. By applying the light cone lemma to the second equation we conclude that

$$
C=\alpha Z, \alpha \neq 0,
$$

plugging it back into the first equation we get

$$
c_{1}(K) \cdot C=\alpha c_{1}(K) \cdot Z=0,
$$

which implies

$$
c_{1}(K) \cdot Z=0,
$$

which is impossible by the choice of $\mathrm{Z}$. This proves Theorem A and therefore Gompf's conjecture.

Theorem A implies the following well known theorem in Kahler surface classification theory [G.H.]. 
Corollary F. (Enriques) Let $M$ be a minimal Kahler surface with $c_{1}(K)^{2}<0$. Then it must be irrational ruled.

From the discussion we also learned that if a symplectic four manifold (with $b_{2}^{+}=1$ ) has a degenerate cup product map on $H^{1}(M, R)$, then its minimal model must satisfy $c_{1}(K)^{2}=0$.

Next let me provide a reasonable interpretation of the argument. In fact the previous argument concerning a family of classes $k \cdot Z$ is very similar to some arguments in the Riemann Roch theorem for surfaces. In fact, quite often algebraic geometers choose some ample class $H$ and study the global sections of the family of line bundles $k \cdot H$ and their asymptotic behaviors in $k$. The only difference here is that we use a class $Z$ with $Z^{2}=0$ (which is not even "big" in terms of the lanquage of algebraic geometers). If one takes Taubes' fundamental theorem " $S W=G r$ " for granted, the remaining argument seems to be even simpler than the corresonding argument in Kahler geometry. Suppose one recalls the standard argument from e.g. Griffiths-Harris [GH]. They first argue that the image of the so called Albanese map is a smooth holomorphic curve imbedded inside the Albanese torus (some quotient of $H_{1}$ ), then by using the Riemann Roch theorem and the local geometry of holomorphic curves, they argued that the regular fibers of this fibration must be complex curves of genus zero. Of course this argument is not expected to apply in the Seiberg Witten proof we offer here. However, it seems that there is still some kind of analogue between these two arguments. The local geometry of the complex curves is replaced by the local geometry of pesudo-holomorphic curves with no essential change. In this case Taubes' fundamental theorem plays the role of the Riemann-Roch theorem. Now the argument of wall crossing formula replaces the role of the Albanese map. In fact the readers may notice that the class which plays the central role in the wall crossing number calculation is the exact same class corresponding to the fiber class of the Albanese map.

Therefore, one can interpret in this way that the very existence or nonexistence of a pesudo-holomorphic curve stable under almost complex perturbation is strong enough (by comparison with the fact that curves generated by the Riemann-Roch theorem may not be stable even under complex deformation) to remedy the defect that in the symplectic category. Even though we don't have a holomorphic Albanese map to control the geometry, its position is weakly replaced by the wall crossing formula [KM,LL1].

As another application of Gompf's conjecture, let us prove the following theorem.

Theorem B. Let $M$ be a $b_{2}^{+}=1$ symplectic four manifold (not necessarily 
minimal). If $c_{1}(K) \cdot \omega<0$, then it must be either rational, rational ruled or irrational ruled.

This theorem means that if we remove the rational or irrational ruled surfaces from the picture, the "ordindary" symplectic four manifolds with $b_{2}^{+}=1$ still satisfy $c_{1}(K) \cdot \omega \geq 0$ [T3].

Unlike the $b_{2}^{+}>1$ case, we can not conclude that $c_{1}(K)$ itself is represented by pseudo-holomorphic curves. Holomorphically (in the cases of Kahler surfaces) we know $p_{g}=\operatorname{dim}_{C} H^{0}(M, K)=0$ or by direct calculation (using the wall crossing formula) we see that $S W(K)$ in Taubes' chamber is 0 .

Proof. First we do the symplectic blow down until M becomes minimal. Notice that if the original manifold satisfies $c_{1}(K) \cdot \omega<0$, then this expression is still negative after the blowing down process. Without loss of generality, we can assume from the very beginning that the symplectic manifold is minimal. Now there are two possiblities. If $c_{1}(K)^{2}<0$, then we know by the affirmative answer of the Gompf's conjecture that the manifold must be irrational ruled. The only case we need to handle is when $c_{1}(K)^{2} \geq 0$. Combining this condition and $c_{1}(K) \cdot \omega<0$ together implies that the class $c_{1}(K)$ is in the backward light cone. Assuming that $b_{2}>1$, we use the trick as in the proof of Gompf's conjecture to find a class $Z$ on the boundary of the forward light cone (assumption $b_{2} \geq 2$ must be used) with $Z \cdot Z=0$ and $Z \cdot c_{1}(K)<0$.

If the first Betti number is zero, then we can prove that the manifold must be a ratoinal or rationally ruled surface. If not, there will be an infinite number of classes whose Seiberg Witten invariants are not zero, which contradicts to the finiteness of the Seiberg Witten basic classes in the metric chambers. From now on, we can always assume that $b_{1}$ is not zero. In this case, the general wall crossing formula [LL1] will be used.

Adapting Taubes' proof [T1] again, one can argue that the cup product pairing on $H^{1}$ is not degenerate. The reason is as follows: If the cup product is degenerate, then the wall crossing numbers for all the $\operatorname{spin}_{c}$ structures are zero. Using Taubes' existence theorem for $S W(K)$ [T2], implies that $c_{1}(K)$ is represented by a symplectic curve. However this contradicts to the fact, $c_{1}(K) \cdot \omega<0$.

Once we know that the cup product pairing is non-zero, the same argument as in the proof of Gompf's conjecture shows us that it must be minimal and irrational ruled. In this case, it must be an irrational ruled surface with an elliptic base.

On the other hand, if $b_{2}$ is equal to one, denote the positive generator of $H^{2}(M, R)$ by $H$, then it is easy to see that $K=-3 H$ in this case. In fact 
the manifold just "looks like" a homology $C P^{2}$. Basically one can use the previous argument with some special care. First blow up one point on the manifold $M$. Now it does have a light cone in $H^{2}(M)$. Using the choice $Z=H-E$ and arguing as before, one can still conclude that the blown up manifold is a rational ruled surface. Therefore, the manifold $M$ must be $C P^{2}$. The subtlety is that the manifold to which we are applying Taubes' theorem " $G r=S W$ " is not minimal any more and a priori the problem of "multiple -1 curves" can happen sometimes. However, the simplicity of the topology actually tells us that for these $k \cdot Z$ of classes, Taubes' theorem " $S W=G r$ " is still correct. The reason is because one can easily argue that the cohomology class $E$ is represented by the unique -1 curve on the manifold (almost by construction). Using the fact that $H^{2}(M)$ is of dimension two and the fact that $E$ appears in $k \cdot Z$ with negative coefficients, one can argue easily that the muliple coverings of -1 curves can never show up and Taubes' theorem remains valid in this case. After this simple digression, one is able to use Taubes' theorem to safely prove the result. This ends the proof of the theorem.

As an application of the above discussion, one can prove the following theorem which was actually the starting point of the whole discussion.

Theorem C. Let $M$ be a symplectic four manifold carrying psc metrics, then $M$ must be some blow up of either rational, rational ruled or irrational ruled surfaces.

With Theorem B at hand, the proof is actually not hard.

Proof. First by Taubes' existence theorem [T2], $S W(K)$ is non-zero. On the other hand, Witten's vanishing theorem [W1,2] tells us that $S W(K)$ is zero for psc metrics. Combining these two facts together implies that $b_{2}^{+}$ must be one. Let us begin the discussion now.

First consider the case $b_{1}>0$. If $b_{1}$ is actually bigger or equal to 4 , then Gompf's conjecture proved above implies that it must be irrational ruled even without using the psc metrics. Therefore, we only need to consider the $b_{1}=2$ case. When $b_{1}^{+}=2$, one can easily calculate $c_{1}(K)^{2}=2 \chi+3 \sigma \leq$ 0 . We can always blow it down to its minimal model. It is easy to see that the minimal model must satisfy $c_{1}\left(K_{\min }\right)^{2}=0$. Otherwise the main theorem will imply that the minimal model must be geometrically ruled with $b_{1} \geq 4$, contradicting the assumption on $b_{1}$. If $M$ itself is minimal, then one can draw the conclusion right away from Lemma 4. One learns that $c_{1}(K) \cdot \omega_{p s c}<0$ and therefore, $c_{1}(K)$ must be on the boundary of the backward light cone. From Theorem B one knows that it must be irrational ruled with an elliptic base. Therefore, $M$ comes from several blowing ups of its minimal model with $c_{1}\left(K_{\text {min }}\right) \cdot c_{1}\left(K_{\text {min }}\right)=0$. Because we don't know 
yet whether the property of carrying psc metrics can be preserved under the blowing down process, we cannot assume that the minimal model of $M$ always carries psc metrics even though $M$ itself does.

Using the general wall crossing formula proved in [LL1] and the existence of the psc metrics, one learns that $c_{1}(K) \cdot C= \pm 2$. On the other hand, the class $C$ comes from the cup product of elements in $H^{1}(M, R)$. Therefore, it must be perpendicular to any cohomology class which is the image of some topological sphere. In particular it is automatically perpendicular to any -1 symplectic curve on $M$. Suppose we have a map from an $S^{2}$ to $M$,

$$
f: S^{2} \longrightarrow M
$$

Then we know that

$$
0=\int_{S^{2}} f^{*}(C)=P D\left(f_{*}\left(S^{2}\right)\right) \cdot C
$$

as $S^{2}$ doesn't have any first homology.

From here one can calculate

$$
c_{1}(K) \cdot C=c_{1}\left(K_{\min }\right) \cdot C= \pm 2
$$

by using the adjunction formula $c_{1}(K)=c_{1}\left(K_{\text {min }}\right)+\sum E_{i}$. This implies that $K_{\text {min }}$ cannot be torsion.

Suppose we have a specific psc metric $g$ on manifold M, then by Lemma $4, c_{1}(K) \cdot \omega_{g}$ must be negative (where $\omega_{g}$ is the self dual harmonic two form assoicated with $g$ ). Let us denote by $\omega_{g}$

$$
\omega_{0}+\Sigma a_{i} \cdot E_{i}
$$

where $\omega_{0}$ is a class in the forward light cone on the minimal model of $M$, and these $E_{i}$ are the disjoint -1 curves we blow down. Now one uses the trick of -1 reflection constructed by Morgan-Friedman [FM]. Namely we can perform the diffeomorphisms on manifold $M$ to switch these $E_{i}$ to $-E_{i}$ without touching the part of the cohomology which comes from the minimal model. The main point is that if we pull back the metric $\mathrm{g}$ by this -1 diffeomorphism, the new metric will become another psc metric. By choosing a suitable diffemorphism, one can arrange the new $\omega_{g}$ such that all the coefficients $a_{i}$ in front of $E_{i}$ are negative. Now we can recalculate $K \cdot \omega_{g}$.

$c_{1}(K) \cdot \omega_{g}=\left(c_{1}\left(K_{\text {min }}\right)+\Sigma E_{i}\right) \cdot\left(\omega_{0}+\Sigma a_{i} \cdot E_{i}\right)=c_{1}\left(K_{\text {min }}\right) \cdot \omega_{0}+\Sigma a_{i} \cdot(-1)<0$. 
However, this implies that

$$
c_{1}\left(K_{\text {min }}\right) \cdot \omega_{0}=c_{1}(K) \cdot \omega+\Sigma a_{i}<0 .
$$

It is obvious that the above inequality implies that $c_{1}\left(K_{\text {min }}\right)$ is lying in the backward light cone of the minimal model. Then Theorem B finishes the job. Therefore, manifold $M$ comes from some blow up of an irrational ruled surface with an elliptic base. This ends the proof when $b_{1} \neq 0$.

From now on let us assume $b_{1}=0$. Now the wall crossing formula of Kronheimer and Mrowka $[\mathrm{KM}]$ is needed in the discussion.

From the proof of the Gompf's conjecture, any symplectic manifold of this type must come from some minimal model which satisfies $c_{1}\left(K_{\text {min }}\right)^{2} \geq$ 0 . Therefore, to show that it is rational or irrational ruled, we must show that $c_{1}\left(K_{\text {min }}\right)$ in fact lies in the backward light cone. Playing the same trick as before, one can always arrange the psc metric $g$ such that

$$
\omega_{g}=\omega_{0}+\Sigma a_{i} \cdot E_{i}
$$

with $a_{i}$ all negative. The same calculation shows that $K_{\min } \cdot \omega_{0}$ is strictly negative. This is exactly what we need in order to show that the rational curve with non-negative self intersection number on the minimal model exists.

The fact that symplectic manifolds with psc metrics can be classified without having a complete classification of the $b_{2}^{+}=1$ symplectic four manifolds gives us several interesting corollaries.

Corollary G. The following three conditions are equivalent for a symplectic four manifold.

1. Having a positive scalar curvature metric.

2. Having a super $P$ cell (the chamber whose $S W$ invariants are all zero).

3. Having a rational curve with non-negative self-intersection number.

From the discussion one learns that the symplectic blowing down process actually preserves the property of carrying psc metrics. It will be very interesting to see whether this property holds for higher dimensional symplectic manifolds.

By combining the following lemma proved by Witten [W],

Lemma 11. (Witten) [W] If a four manifold carries non-negative scalar curvature metrics, then one of the two possibilities happens.

1. It has a super $P$ cell (which it means that the Seiberg Witten invariants

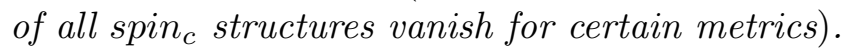

2. It is Kähler with a Kähler scalar flat metric. In this case they are either K3, Enriques surfaces, $T^{4}$, or rational or irrational ruled surfaces.

Now one can classify the symplectic manifolds with non-negative sclar curvature metrics. 
Corollary H. Let $M$ be a symplectic four manifold with non-negative scalar curvature metrics, then it must be either rational, rationally ruled, irrationally ruled, Enriques surfaces, $T^{4}$ or K3 surfaces. In particular all these symplectic four manifolds carry Kähler structures.

In fact, the above classification of symplectic manifolds with psc metrics gives us a more elegant proof of the fact that symplectic structures of the rational or irrationally ruled manifolds are in fact unique up to deformation. Unlike the previous proof [LL2], the above argument does not contain any topological assumption of $M$ at all. Notice that in the previous argument [Liu] the classification of symplectic manifolds with the psc metrics was done by doing calculations using the Hasse-Minkowski classification of the quadratic forms and then finding out some explicit special cohomology class to generate the rational curve we needed. In the current revised version of the paper that kind of messy argument is completely replaced by the simpler argument given above.

As another application of Gompf's conjecture, one can actually rederive the previous result [LL2] about the nonexistence of square zero spheres in the $b_{2}^{+}=1$ symplectic four manifold $M$. Based on the similar idea of the proof of the Gompf's conjecture, one can show the following:

Theorem D. [LL] Let $M$ be a $b_{2}^{+}=1$ symplectic manifold. If $C$ is a class such that $C \cdot \omega>0$ with $C^{2}=C \cdot c_{1}(K)=0$, then $C$ can not be represented by a square zero sphere.

Remark. This theorem is a key step in proving the adjunction inequality which was argued along a different method before. One can actually formulate a general statement without using the fact that $M$ is symplectic. However as we only have the existence result within the symplectic category [T2], let us limit ourself to this category.

Proof. With the main theorem of this paper at hand, one can consider two cases. First we consider the case that $c_{1}(K) \cdot \omega \geq 0$, otherwise $M$ has to be rational or irationally ruled. As the manifolds in the second case always carry the psc metrics, we will merely use the fact that it is a symplectic manifold carrying psc metrics.

In either case, let us blow up a point on the manifold $M$. Now one can make sure the $c_{1}(K)$ of this symplectic structure is non-torsion. Then by a small perturbation of $\omega$ we can assume $c_{1}(K) \cdot \omega$ to be non-zero.

In the first case the $c_{1}(K) \cdot \omega$ must be greater than zero or it will contradict Theorem B. From this we know that for some metric chamber with $c_{1}(K) \cdot \omega_{g}>0$, the $S W(K)$ is equal to \pm 1 .

Now consider the -1 reflection $[\mathrm{FM}]$ generated by the -1 sphere representing $E_{n}=E \pm n C$, where $n$ ranges over all integers. Using the fact 
$c_{1}(K) \cdot C=0$, One learns that

$$
\operatorname{Re}_{E_{n}}\left(c_{1}(K)\right)=c_{1}(K)-2 E_{n}=c_{1}(K)-2 E-2 n C
$$

and we also know that

$$
R e_{E_{n}}\left(c_{1}(K)\right) \cdot R e_{E_{n}}\left(\omega_{g}\right)>0
$$

if

$$
c_{1}(K) \cdot \omega_{g}>0
$$

i.e. $K$ is a basic class for the metric g. On the other hand the $S W$ invariants are diffemorphism invariants up to chambers. This implies that for any generic smooth metric $h$ we pick, if the cup product $\operatorname{Re}_{E_{n}}\left(c_{1}(K)\right) \cdot \omega_{h}>$ 0 , then $\operatorname{Re}_{E_{n}}\left(c_{1}(K)\right)$ is a basic class of $h$. As in the proof of Gompf's conjecture, this creates a contradiction. For any metric $h$ we choose,

$$
\operatorname{Re}_{E_{n}}\left(c_{1}(K)\right) \cdot \omega_{h}=\left(c_{1}(K)-2 E-2 n C\right) \cdot \omega_{h}>0
$$

for sufficiently negative $n$. We argue by combining our assumption and the light cone lemma that $C \cdot \omega_{h}>0$. The fact that there is an infinite number of basic classes for $h$ contradicts the finiteness of the basic classes for a fixed metric.

On the other hand, if $M$ carries psc metrics. We always know that $c_{1}(K)$. $\omega_{p s c}<0$. Doing the same refletion one gets $\operatorname{Re}_{E_{n}}\left(c_{1}(K)\right) \cdot \operatorname{Re}_{E_{n}}\left(\omega_{g}\right)<0$ for any $g$ whose scalar curvature $R_{g}$ is positive. As $R e_{E_{n}}$ is realized by a diffemorphism which certainly moves psc metrics to psc metrics, these $\operatorname{Re}_{E_{n}}\left(c_{1}(K)\right)$ have trivial $S W$ invarints in the negative chamber. By letting $n$ be sufficiently negative, its cup product with any period of psc metrics is essentially positive. This tells us that for these special $n$, there are different psc metrics sitting on the both sides of the walls. This forces the wall crossing number of these classes to be zero. On the other hand as the cohomology classes come from pulling back the $c_{1}(K)$ by some diffemorphisms, they must share the same wall crossing number with $c_{1}(K)$. (which is definitely non-zero by combining Witten's vanishing result [W] and Taubes' existence result [T2].) This proves that in either case the class $C$ cannot exist on the original manifold $M$.

Remark. In fact, the above proof has an analogue in the $b_{2}^{+}>1$ category $[\mathrm{SF}]$ by using a similar idea as in the proof of Gompf's conjecture discussed above. Therefore the above proof of Therorem $\mathrm{K}$ can be viewed as the extension of their result to the $b_{2}^{+}=1$ case. 
As the proof of Theorem E is a modification of Friedman-Morgan's argument, we leave it to the readers as an interesting exercise.

Remark. Theorems B and C suggest that we should view the rational, rational ruled and irrational ruled surfaces as a special category, characterized by the property of carrying positive scalar curvature metrics. Several theorems $[\mathrm{LL} 2, \mathrm{FM}]$ in symplectic geometry hold for the "normal" symplectic four manifolds which satisfy $c_{1}(K) \cdot \omega \geq 0$, but they do not hold for these rational or ruled manifolds. Theorem $\mathrm{C}$ tells us that from the point of view of differential geometry, the reason that these "exceptional" symplectic four manifolds do not satisfy these theorems traces back to the existence of the psc metrics.

Remark. Using the wall crossing formula [LL1] and its slight generalization [LL4] (when the ring pairing on $H^{1}$ is degenerate, one needs to consider some slightly more general Seiberg Witten invariants which have nontrivial wall crossing number), one can easily show that all the $b_{2}^{+}=1$ sympletic four manifolds are not of Gromov simple type. Not only is it true, in fact, there are an infinite number of classes with positive moduli space dimensions which support nontrivial Gromov invariants. The classes $Z$ must satisfy four conditions.

1. $Z^{2}-Z \cdot c_{1}(K)>0$

2. $Z^{2} \geq 0$

3. $\left(c_{1}\left(K^{-1}\right)+2 Z\right) \cdot \omega>0$

4. $c_{1}\left(K^{-1}\right)+2 Z$ is not one of the finite number of basic classes in the positive chambers.

This tells us that the theorem " $S W=G r^{\prime}$ behaves exactly like the Riemann-Roch theorem in complex surface theory. The existence of the ample families of symplectic curves in the symplectic "curve cone" is an exact analogue of the similiar phenomenon happening for holomorphic curves in the "curve cone" of algebraic surfaces, as is guaranteed by the surface Riemann-Roch theorem.

\section{Acknowledgements}

I would like to thank Professor Yau for his guidance in the past several years. I would like to thank Professor Taubes for his encouragement and his patience in listening to my report and reading the draft version of this paper. I would also like to thank T. J. Li for some useful discussion. Theorem E was discovered jointly with him. His suggestions also contributed to the present argument of the Theorem C. 


\section{References}

[FM] R. Friedman and J. Morgan, Algebraic surfaces and SW invariants, Preprint (1995).

[G] L. Gottsche, Wall crossing formula for Donaldson invariants, Preprint (1995).

[GH] P. Griffith and J. Harris, Principles of algebraic geometry, John Wiley \& Sons.

[Gom] R. E. Gompf, A new construction of symplectic manifolds, Preprint.

[KM] P. Kronheimer and T. Mrowka, The genus of imbedded surfaces in the projective space, Math. Res. Lett. 1 (1994), 797-808.

[Liu] A. Liu, Some new applications of general wall crossing formula (early version), Preprint (1995).

[LL1] T. Li and A. Liu, General wall crossing formula, Math. Res. Lett. 2 (1995), $797-810$

[LL2] _ Symplectic structure on ruled surfaces and gneralized adjunction formula, Math. Res. Lett. 2 (1995), 453-471.

[LL3] _ Unpublished note on $b_{2}^{+}=1$ symplectic four manifolds (1995).

[LL4] Unpublished note on the slight generalization of wall crossing formula (1995).

[M] D. McDuff, The structures of rational and ruled symplectic four manifolds, J. Amer. Math. Soc. 1 (1990), 679-710.

[Ser] J.-P. Serre, A course in arithmetic, Springer-Verlag, New York, 1973.

[T1] C. H. Taubes, $S W \rightarrow G r$, Preprint (1995).

[T2] $\quad$ The $S W$ invariants and symplectic forms, Math. Res. Lett. 1 (1994), 809-822.

[T3] More constraints on symplectic manifolds from SW equations, Math. Res. Lett. 1 (1995.), 4-9.

[T4] Counting of pseudo-holomorphic tori, Preprint (1996).

[T5] $\quad G r \rightarrow S W$, Preprint (1996).

[T6] The Seiberg Witten invariants and the Gromov invariants, Math. Res. Lett. 2 (1995), 221-238.

[W] E. Witten, Talks at MIT and Harvard (1994).

[W1] _ Monopoles and Four Manfolds, Math. Res. Lett. 1 (1994), 769-796.

[Wil] H. Wilson, Calabi-Yau three folds with large Picard number, Invent. Math. 98 (1989), 139-155.

Department of Mathematics, Harvard University, Cambridge, M A 02138

E-mail address: akliu@math.harvard.edu 